\title{
Live Mapping of Crystalline Regions During in-situ Heating (TEM and STEM)
}

Benjamin Miller ${ }^{1}$, Anahita Pakzad ${ }^{2}$, Liam Spillane $^{3}$, Bernhard Schaffer ${ }^{2}$ and Cory Czarnik ${ }^{2}$

${ }^{1}$ Gatan Inc., California, United States, ${ }^{2}$ Gatan Inc., United States, ${ }^{3}$ Gatan Inc., Pleasanton, California, United States

During in-situ experiments it is often important for the user to make good decisions about what to do next. Should the in-situ conditions be changed, or should the data collection frequency and details be modified? Has the sample been transformed during the experiment, or destroyed, or is it unchanged? In some cases, a simple visual inspection of the live 2D image is sufficient to answer these questions and make good decisions. In other cases, the decision is not so straightforward, and some processing is required before the user can draw any conclusions. Commercial software can already do some processing to transform the raw sensor output into "clean" data, or to produce a live FFT. However, new data acquisition and processing methods are constantly being developed, and often the built-in live processing in commercial software does not yet support these advances. Gatan Microscopy Suite (GMS) has always had scripting capabilities which can be used to customize live processing, and recently Python scripting has been added, making this more accessible to the larger community.

In this work we demonstrate real-time Python processing of both 4D STEM datasets and high-resolution images to map the spatial distribution of crystallinity during acquisition. It is inherently difficult to see anything in the raw data of a series of 4D STEM datasets due to the dimensionality, which prohibits direct display on a 2D monitor. It can also become difficult to see the crystallinity of a material over a large field of view because modern cameras, like the K3 IS used to collect this data, produce images that are too large to display on most monitors.

The methodology for this live Python-based processing is shown in Figure 1. In the case of 4D STEM, each diffraction pattern is processed to find the maximum intensity pixel, while masking a user-defined central region of the pattern. The direction from the pattern center, spacing, and intensity of this maximum point are determined, and color maps are produced. The hue can be either the angle or distance from center while the brightness of the color is determined by the intensity. A 2D color map is thus generated from each 4D data cube. If a series of data cubes is acquired, a map can be generated for each one, resulting in a color video.

This approach can be extended for processing large high resolution TEM images. First a grid of overlapping windows is extracted from a single large 2D image, and the FFT of each window is computed. These FFTs are placed into a 4D cube which is analogous to that generated using 4D STEM, but with diffractograms instead of diffraction patterns. This 4D cube of FFTs can then be analyzed in the same way as a 4D STEM data cube. This processing has been applied during video recording of Sn nanoparticle melting and recrystallization, where the temperature was oscillated between 50 and $300{ }^{\circ} \mathrm{C}$. Maps processed after acquisition are shown in Figure 2, and screenshot videos of live processing during acquisition will be shown in my talk. While it was not obvious from the raw video that the nanoparticles re-crystallize in different orientations each time, this is clear from the maps.

Figures 1 and 2 primarily demonstrate the parallels and similarities between the 4D STEM and HR TEM based approaches. However, there are differences and tradeoffs which would make one or the other optimal for a given experiment. The primary benefit of the FFT-based approach is the potential for capturing data with the high temporal resolution afforded by TEM and modern in-situ cameras. The diffractogram data is also easier to process since the center of the pattern is always in the exact center of the image. However, the spatial resolution of the FFT-based maps tends to be poor and the volume of reciprocal space that is covered is usually less than with 4D STEM. Finally, the FFT approach is better 
suited to microscopes that are optimized for TEM imaging, ideally with aberration correction. These benefits and limitations will be discussed in my presentation.

4D STEM:
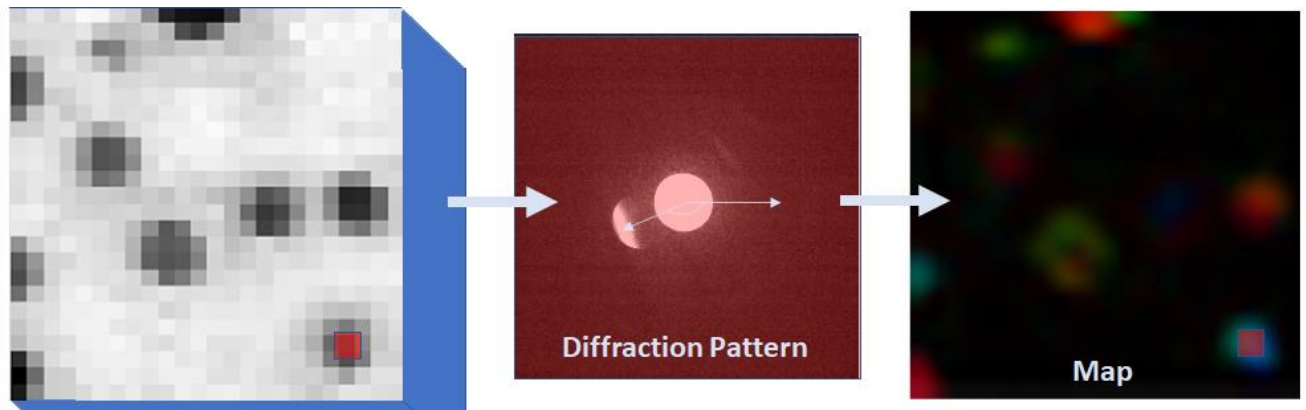

HR TEM
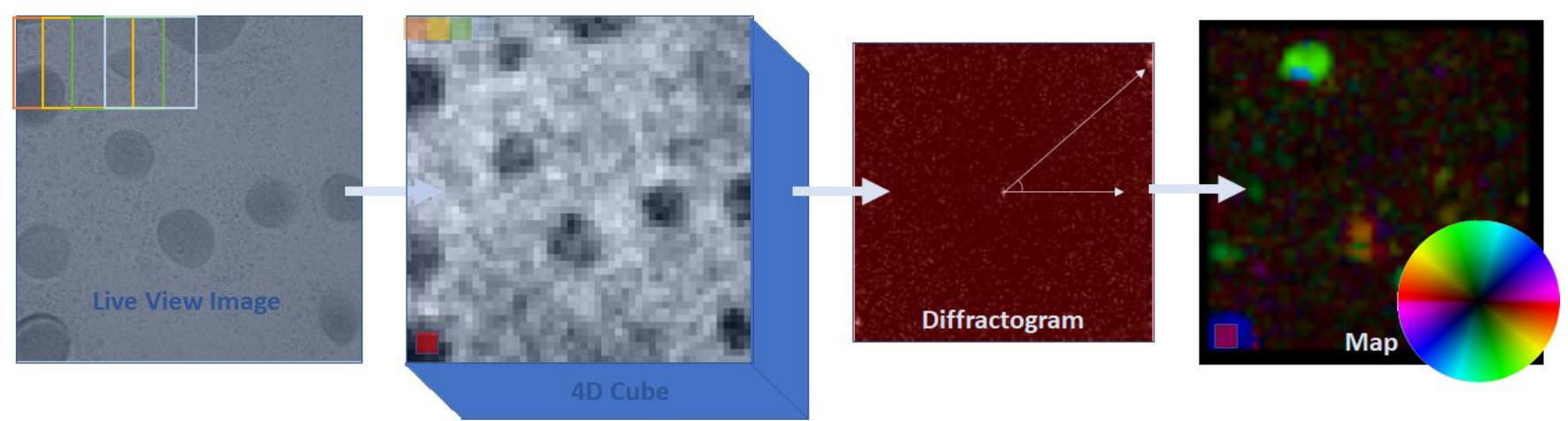

Figure 1. Figure 1 Live Processing Methodology. Top: 4D STEM data cube is processed to find the brightest pixel in each pattern. Values of the intensity and position are then used to generate a color map. Bottom: High resolution TEM image is split into overlapping windows, and FFTs computed to generate a 4D data cube which is then processed in the same way to generate a color map. 

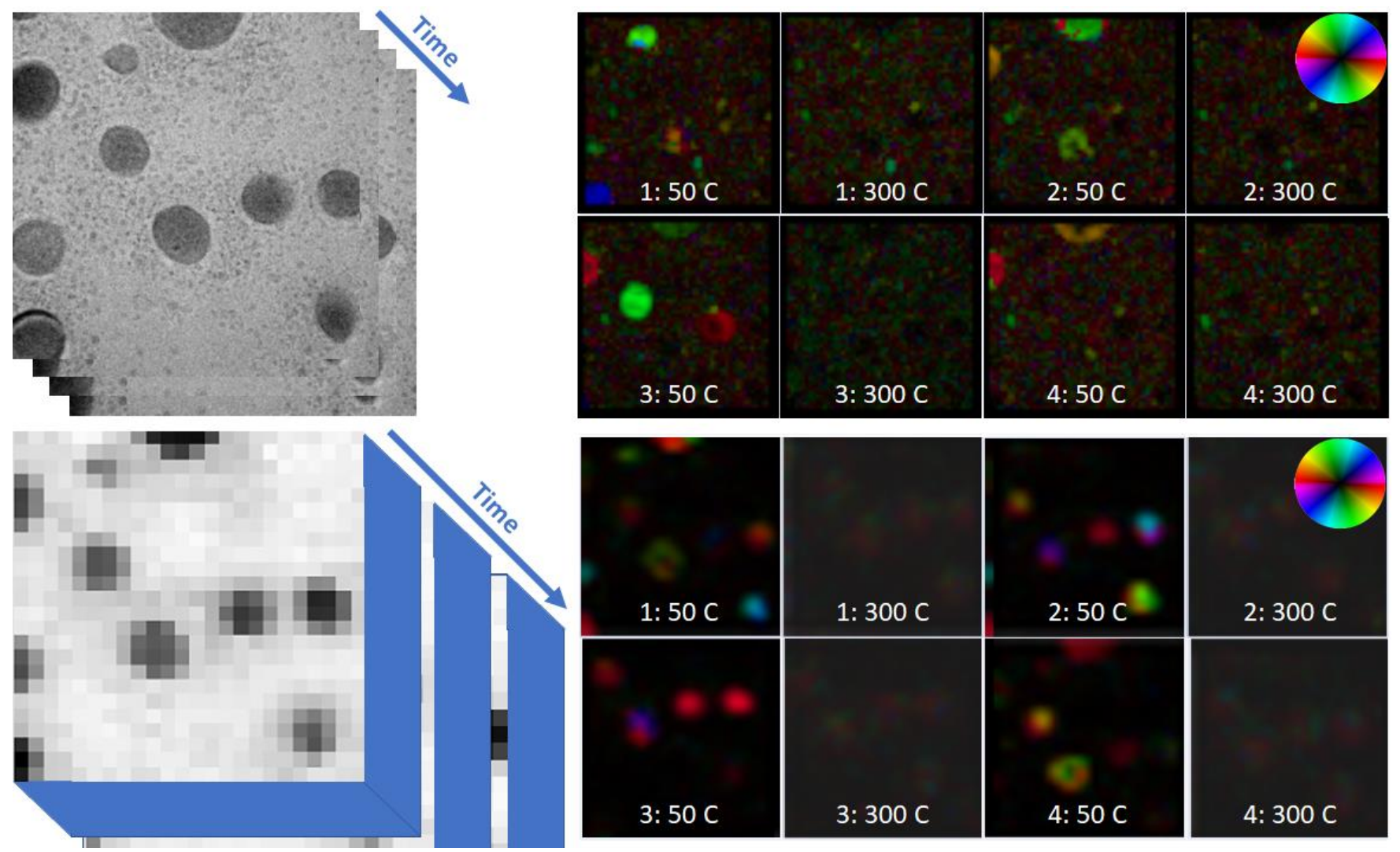

Figure 2. Figure $2 \mathrm{Sn}$ nanoparticle video and 4D STEM time series capturing repeated melting and recrystallization. Both sets of data were acquired from the same region of the sample on the same microscope using a K3 IS camera (TEM) and Continuum IS camera (4D STEM) and were each processed to produce a series of similar maps. Maps from 4 cycles in each case show that particles recrystallize in different orientations each cycle. 